\title{
Entre " jouer avec » l'argent et " parier pour » l'argent : une analyse sociologique du double statut de l'argent du jeu en Chine
}

\section{Shen $\mathrm{Hu}$}

\section{OpenEdition}

Journals

Édition électronique

URL : http://journals.openedition.org/sdj/415

DOI : $10.4000 /$ sdj. 415

ISSN : 2269-2657

Éditeur

Laboratoire EXPERICE - Centre de Recherche Interuniversitaire Expérience Ressources Culturelles Education

\section{Référence électronique}

Shen $\mathrm{Hu}$, «Entre « jouer avec » l'argent et « parier pour » l'argent : une analyse sociologique du double statut de l'argent du jeu en Chine », Sciences du jeu [En ligne], 3 | 2015, mis en ligne le 22 juillet 2015, consulté le 20 avril 2019. URL : http://journals.openedition.org/sdj/415 ; DOI : 10.4000/sdj.415

Ce document a été généré automatiquement le 20 avril 2019

Tous droits réservés 


\title{
Entre " jouer avec " l'argent et " parier pour " l'argent : une analyse sociologique du double statut de l'argent du jeu en Chine
}

\author{
Shen $\mathrm{Hu}$
}

1 Les joueurs de jeux d'argent ont longtemps subi une stigmatisation scientifique. Cette dernière, dans la lignée de l'approche marginaliste de Jeremy Bentham, consiste à dénoncer l'« irrationalité économique» d'une population de joueurs censés, théoriquement, perdre plus qu'ils ne gagnent ${ }^{1}$. Une telle dénonciation repose sur un présupposé que les joueurs sont systématiquement mus par l'appât du gain. Or, comme l'a souligné Guy Carcassonne avec peut-être un peu d'excès de certitude, "Rien n'est plus faux, en effet, que de croire que le jeu est d'abord une question d'argent. Non, le jeu est d'abord une question de jeu » (Carcassonne, 2011, p. 20).

Alors que la remarque de ce juriste ne s'est pas accompagnée d'une démonstration empirique, ce fut Jean-Pierre Martignoni qui a essayé de dédramatiser l'argent comme la raison unique de jouer, en se rendant dans des espaces réels de jeux. Mais après avoir indiqué que l'argent, une fois misé, peut devenir « instrument au service d'une pratique » (Martignoni, 1993, p. 207) et que « le joueur ne joue pas que [souligné par l'auteur] pour l'argent» (p. 211), le sociologue a tout de même préféré prévenir ses lecteurs que les discours de ses interviewés pourraient comporter « diverses interférences : infatuation, occultation, exagération, égotisme... » (p. 39). Cette réserve qu'a exprimée Jean-Pierre Martignoni ne devrait pas être sans rapport avec son regard uniquement porté sur les joueurs participant à des jeux d'argent mono-joueurs (pari hippique, loterie, machine à sous). Car, ce faisant, il n'a pu procéder qu'à une analyse micro-individuelle des joueurs, dont le système interprétatif est peu capable de mettre en évidence des déterminants supra-individuels. 
Cherchant à apporter de nouveaux éléments empiriques à partir d'un autre système interprétatif fondé, quant à lui, sur l'identification des contraintes sociales d'interaction, nous nous sommes intéressés au rapport à l'argent de joueurs chinois qui participaient à des jeux multijoueurs ${ }^{2}$.

4 Les données empiriques ayant alimenté notre analyse sont issues d'une enquête de terrain que nous avons réalisée en 2012 dans quatre grandes villes chinoises: Pékin, Shanghai, Xiamen et Qingdao. Ces dernières ont été sélectionnées pour une raison de facilité de recrutement d'interviewés. Vingt-sept entretiens semi-directifs ont été réalisés auprès d'une population diversifiée selon des critères socio-démographiques, tels que le sexe, l'âge, le niveau d'instruction, la profession et la situation matrimoniale. En incluant un questionnement focalisé sur les expériences de jeux d'argent multijoueurs de nos interviewés, l'enquête comportait pourtant une problématique plus large sur leur rapport à l'argent dans la vie quotidienne en général. Le rapprochement que nous avons établi entre ces deux thématiques se justifie par notre hypothèse de départ, selon laquelle l'usage de l'argent est socialement encastré dans les liens sociaux plus ou moins affectifs des individus, et que ce mécanisme d'encastrement social de l'argent préside également aux jeux d'argent multijoueurs.

5 Cette hypothèse renvoie au concept de "marquage social de l'argent» formulé par Viviana A. Zelizer. Avec l'approche interactionniste qu'il requiert, le « marquage social de l'argent » est selon nous l'un des outils analytiques les plus aptes à mettre en évidence qu'« [i]l n'y a pas d'argent unique, uniforme et généralisé, mais des monnaies multiples : les êtres humains marquent les diverses devises en les rapportant à de nombreux types d'interactions sociales (à tous, peut-être), exactement comme ils créent des langages appropriés à tel ou tel contexte social» (Zelizer, 2005, p. 52). Les jeux d'argent multijoueurs constituant un espace d'interaction sociale, notre étude a mis en évidence la façon dont, selon la nature du lien social entre les joueurs, le rôle de l'argent du jeu oscille entre l'argent comme instrument ludique et l'argent comme objectif ultime.

\section{Condamnations éthico-juridiques du « pari » en Chine}

6 Avant de procéder à la démonstration concrète du double statut de l'argent du jeu, nous estimons qu'il est judicieux de nous arrêter sur un contexte culturel chinois qui touche de près à notre sujet.

7 En effet, notre étude sur le marquage social de l'argent du jeu a largement été facilitée par la distinction lexicale, dans la langue chinoise, entre «玩wán» (qui veut dire «s'amuser») et «奢dǔ » (qui veut dire "parier»). Les significations que les Chinois accordent à chacun de ces deux mots correspondent parfaitement à la dichotomie théorique, élaborée par André Neurisse, entre «jeu » et «pari». Selon cet économiste, "Lorsqu'on se réfère à la nature de l'événement, le jeu se définit comme la participation volontaire de deux ou plusieurs personnes à une même occupation; le but en est le divertissement et, accessoirement ou principalement, le profit, lorsqu'il y a offre d'un prix. Quant au pari, il peut s'analyser comme un accord d'offrir un prix à celui qui a prévu l'arrivée d'un événement ; c'est un quasi-contrat, dont le but est de ratifier une opinion contestée par autrui » (Neurrisse, 1993, p. 18).

8 Si les Chinois n'ont pas besoin d'une théorie pour saisir cette dichotomie qui est, pour eux, une évidence, c'est probablement parce qu'ils ont intérêt à ne pas confondre les deux 
activités; le «pari pour l'argent », contrairement au «jeu avec l'argent », faisant l'objet, dans la société chinoise, d'une double condamnation éthique et juridique.

Sur le plan éthique, la condamnation renvoie d'abord à ce que Roger Caillois a considéré comme une "opinion unanime", laquelle "admet comme une évidence qui ne souffre même pas contestation, que le travail, le mérite, la compétence, et non le caprice du coup de dés, sont les fondements à la fois de la justice nécessaire et de l'heureux développement de la vie collective». Et l'auteur a tout à fait raison de rajouter que "l'idéal communiste de l'administration des sociétés porte ce principe à l'extrême " (Caillois, 1991, p. 304-305).

10 La raison qui dissuade les «joueurs " chinois de passer pour des "parieurs » peut également se rapporter à un principe de l'économie domestique, lequel condamne toute forme de dilapidation de l'argent dont le pari fait partie. Si un tel principe se dote d'un caractère transculturel (Amadieu, 2011), il semble prendre la forme, dans la société chinoise, autant d'une vertu idéologique que d'un habitus pratique ${ }^{3}$. Dans cette perspective de frugalité vertueuse, ce serait une dilapidation « immorale » que d'investir dans un «quasi-contrat de pari », dont l'avantage financier n'est réservé qu'aux parieurs les plus performants. Or, dans un «jeu» dont le but ultime est le divertissement, l'éventuelle perte pourrait être interprétée comme un simple prix à payer, au même titre, par exemple, que l'achat d'un billet d'entrée donnant accès à un parc d'attraction.

11 Enfin, sur le plan juridique, l'élément qui conduit le plus sérieusement les Chinois à se classer parmi les «joueurs» réside dans l'article 303 du code pénal chinois en vigueur: "L'organisation du pari mutuel à but lucratif, la tenue d'une maison de pari et l'exercice d'un métier de parieur sont punis de trois ans maximum d'emprisonnement, d'incarcération ou de mise sous surveillance. Une amende y est également associée ». En effet, tout en lui refusant le droit de "parier pour l'argent », cette loi a accordé au peuple chinois la permission juridique de « jouer avec l'argent ».

\section{« Jouer » avec ses proches}

12 Il n'est donc pas étonnant que les joueurs chinois ont tenu à nous préciser, lors de nos échanges, que c'était le "jeu », et non pas le "pari », auquel ils s'étaient livrés. Et force est de constater que, pour nous en persuader, beaucoup d'entre eux précisaient que c'était avec leurs parents ou leurs amis qu'ils avaient «joué » (et non parié) de l'argent. Sans exclure la possibilité qu'il s'agisse là d'une manière artificielle de se conformer aux normes sociales que nous venons d'aborder, nous estimons qu'il est socialement difficile pour un individu de convoiter l'argent de ses proches, notamment dans le cadre d'une activité non vitale comme le jeu (il est vrai que la question sera moins évidente à propos, par exemple, de l'héritage, source importante de contentieux familiaux).

13 En effet, dans la mesure où le pari est un contrat financier dont l'objectif final de chaque signataire est de tirer profit des mises des autres signataires, ce principe entre immédiatement en contradiction avec le lien parental ou le lien amical. Car ces derniers relèvent également d'un contrat, mais un contrat affectif qui repose sur le principe de désintéressement financier. Plus précisément, la souscription d'un contrat de pari, par des personnes déjà engagées dans un contrat affectif, risque grandement d'entraîner la résiliation de ce dernier; et, inversement, pour préserver leur lien affectif, il leur est conseillé de renoncer à la souscription de tout contrat de pari (ou, au moins, de réussir à 
dissimuler l'aspiration financière, ce qui est pourtant difficile compte tenu du paradoxe entre la somme d'un gain désirable et la suspicion que ce gain désirable pourrait facilement susciter).

14 Ceci dit, le désintéressement que stipule le contrat affectif ne suppose aucunement l'interdiction normative de l'exercice de jeux d'argent entre personnes proches; seulement, afin de rester dans la sphère du « jeu » tout en s'écartant du «pari », on sera amené à respecter le principe de don réciproque, lequel sera concrétisé par l'obligation, imposée au gagnant, de restituer ses gains auprès du perdant :

Mon gendre est très respectueux. Quand il gagne de l'argent de ma part, il donnera cet argent à ma fille pour qu'elle me le rende discrètement [...] [Q: Pourquoi discrètement ?] [...] Car s'il me rendait de l'argent devant tout le monde, ce serait comme si on n'était pas en train de jouer de l'argent. (Enquêtée, 55 ans)

La raison de choisir cette illustration parmi de nombreuses autres réside dans le fait qu'elle permet de révéler un double paradoxe.

D'abord, si le gagnant se doit de restituer ses gains, cet acte risque de faire perdre au jeu d'argent son sens. C'est ainsi que le gendre de l'enquêtée avait dû rendre ses gains de façon discrète, afin que le sens du jeu soit au moins préservé à l'égard des autres joueurs.

Le deuxième paradoxe renvoie au mécanisme même du don réciproque. En effet, une restitution immédiate du gain risque d'être codée comme un acte de "rendre »; alors que, selon la juste remarque de Maurice Godelier (1996), pour maintenir un lien affectif qui est aussi un "rapport de dépendance réciproque ", il faut "re-donner » au lieu de «rendre ». Et si cette nécessité de « re-donner » est beaucoup moins coercitive dans le cadre du lien de parenté relativement solide (voici pourquoi le gendre a encore préféré faire appel à son épouse pour "rendre " ses gains à sa belle-mère), elle devient plus problématique lorsqu'il s'agit de restituer ses gains auprès d'un ami.

C'est, en effet, ce double paradoxe qui permet de rendre intelligible notre constat que, quand il s'agit de jouer entre amis ou en famille, les mises sont généralement très modestes. Car, dans ce cas précis, les gains maximums à empocher seront, a priori, tellement dérisoires que leur non-restitution aura peu d'impact sur la relation sociale des joueurs. Ceci étant, une telle solution ne fonctionne que partiellement, puisque les petits gains peuvent s'accumuler chez un joueur techniquement très compétent ou, ce qui est tout à fait possible, très chanceux. Dans cette perspective, se pose toujours la question de résoudre le double paradoxe; et une autre solution possible renvoie au processus de marquage social de l'argent, lequel consiste pour le gagnant à restituer ses gains au moyen de l'offre de cadeaux (c'est-à-dire de re-donner au lieu de rendre) et, ce, de manière différée afin de ne pas invalider les résultats du jeu en question, ni commettre «le comble de l'impolitesse » que serait un « retour immédiat » (Colson 1962, cité par Testart, 2001, p. 729) :

Une fois notre jeu terminé, chacun va dire combien il a gagné ou perdu, histoire de faire un bilan. Parce que, si une personne a beaucoup gagné, la prochaine fois, elle doit offrir un repas au restaurant à tous les autres. C'est en fait une règle conventionnelle entre nous [...] Normalement, chaque fois que les gains de l'un d'entre nous dépassent 100 yuans [environ 12 euros], celui-ci doit nous inviter au restaurant. (Enquêté, 62 ans) 


\section{«Parier » contre les inconnus}

19 Dans le cadre de notre enquête, nous constatons que l'obligation de restitution du gain amène certains joueurs à se persuader qu'ils auront autant de pertes que de gains, tant qu'ils ne jouent qu'avec leurs amis, et que leur équilibre financier sera fortement menacé dès qu'il y aura la présence d'un inconnu :

Grosso modo, il n'y a pas de grand écart entre mes gains et mes pertes. Par exemple, aujourd'hui je gagne 100 yuans, demain je perdrai 120, puis après demain je me referai. Donc je ne perdrai pas d'argent. Par contre, il faut absolument garder le même groupe, car sinon, tu n'arriveras plus à avoir cet équilibre. Parce que si vous intégrez un inconnu, celui-ci ne reviendra plus, une fois qu'il aura gagné. (Enquêté, 55 ans)

L'inconnu, tel est le type de rapport social qui s'adapte mieux au contrat de pari. Et Georg Simmel n'a pas tort de concevoir une inséparabilité historique entre « le pur commerce de l'argent » et les "étrangers »: "Le partenaire le plus indiqué pour le commerce de l'argent - où cesse, comme on l'a dit à juste titre, l'affectivité - est la personnalité qui nous est intérieurement tout à fait indifférente et qui ne se trouve engagée ni pour nous ni contre nous » (Simmel, 2007, p. 268).

21 Cette concordance entre l'inconnu et le pari est d'autant plus manifeste que les joueurs (au sens de ceux qui cherchent avant tout à se divertir) peuvent être réticents à se confronter à des inconnus; et qu'inversement, pour impliquer ces joueurs dans un pari, les parieurs inconnus savent qu'il est préférable qu'ils soient invités par leurs propres amis, que ces derniers ignorent ou non une telle « intrigue» :

En 2006, je me suis fait escroquer de 4 millions de yuan [environ 570000 euros, selon le taux de change actuel]. C'était à cause d'un pari. En fait, un jour, un de mes meilleurs amis m'avait invité à jouer aux cartes, mais avec des gens que je ne connaissais pas. Le premier jour j'avais perdu 700000 yuans, deuxième jour 800000 yuans, et ainsi de suite jusqu'à ce que je n'ai plus rien. Ce n'était qu'à la fin que je m'étais rendu compte que c'étaient des escrocs et qu'ils avaient un vêtement spécifique pour gagner à coup sûr. [...] Mais en fait, mon ami n'était pas leur complice, c'était son petit frère qui voulait me piéger. Pendant ces jours-là, mon ami avait même essayé de m'empêcher de continuer, quitte à me taper. Sauf que là, tu n'arrivais à écouter personne, car la seule chose que tu voulais, c'était de te refaire afin de terminer le jeu. [...] Aujourd'hui, je garde toujours contact avec cet ami, car je sais qu'il ne l'avait pas fait exprès. Par contre, il se sent toujours coupable vis-à-vis de moi. (Enquêté, 35 ans)

L'histoire racontée par cet enquêté nous montre le risque pour les amis de s'engager dans un contrat de pari, pis, dans un contrat de pari «sournois ». Mais au-delà de cet impact du pari sur la stabilité de la relation amicale, l'histoire met également en lumière un autre risque inhérent au pari entre inconnus. Puisque face à des personnes qui lui sont désormais " intérieurement tout à fait indifférente(s) », la seule possibilité pour le parieur (ou joueur devenu parieur) de se refaire en cas de perte sera de prolonger le contrat de pari jusqu'à ce que l'un décide d'y mettre fin ; or, en général, cette résiliation n'aura lieu que lorsqu'un parieur a suffisamment gagné ou perdu.

Ce constat nous amène à remettre en question une pratique interprétative dominante dans les études sur le jeu, laquelle consiste à imputer systématiquement la ruine du joueur d'argent au mécanisme psychopathologique de l'addiction. En effet, parallèlement à ladite pathologie addictive dont nous ne nions pas la possibilité d'existence, il existe 
pourtant ce mécanisme socio-logique de "se refaire » déclenché par l'«impitoyabilité » des parieurs inconnus. Pour le dire de façon métaphorique, si le pari est un "quasicontrat", sa souscription par un groupe d'inconnus risque d'en faire une sorte de « contrat à durée indéterminée ».

\section{Enjeu matériel et enjeu immatériel}

Si le principal objectif de notre démonstration est la mise en évidence de la manière dont le rôle de l'argent du jeu est socialement encadré par des contrôles sociaux exercés en parallèle $\mathrm{du}$ fonctionnement technique $\mathrm{du}$ jeu, nous estimons qu'il est également intéressant d'aborder un élément inhérent au jeu, lequel pourra conduire de façon intrinsèque les joueurs à se désintéresser de l'argent en tant qu'objectif final.

À ce propos, il faut noter que Guy Carcassonne a déjà fait une remarque intéressante. Selon lui, «De même peut-on jouer au poker pour des pois chiches, et beaucoup débutèrent ainsi, mais ça lasse assez vite. Quant à regarder une bille tourner dans un cylindre, même avec une sonorité inimitable, ou glisser inlassablement des jetons dans un appareil électronique rutilant, même un insensé ne songerait pas un instant à le faire, n'était l'enjeu qui s'y attache. Il est donc d'argent. Mais pourquoi ? Tout simplement faute de mieux.» (Carcassonne, 2011, p. 19)

De là, nous pouvons déduire, sans trop de risque d'erreur, que, si les regards de nombreux chercheurs se focalisent sur l'argent en tant qu'objectif final du jeu, c'est aussi parce que leur répertoire de jeux d'argent est principalement composés de jeux où l'argent, «faute de mieux », est le seul enjeu intéressant.

Sans doute, il n'y a pas de jeu sans enjeu; en revanche, il peut y avoir des enjeux sans argent. Nombreux sont en effet les jeux qui, tout en autorisant l'intervention du caprice du hasard et celle de l'argent, se caractérisent par une technicité si importante qu'on aura du mal à croire que le «faible » puisse battre le «fort ». Et ce sont également des jeux grâce auxquels les joueurs ont plus de facilité pour justifier que ce n'est pas (du moins pas uniquement) pour l'argent qu'ils s'y livrent :

J'adore jouer au mah-jong, surtout le mah-jong à la shanghaienne qui est très amusant. Parce que dans le mah-jong à la shanghaienne, tu ne pourras gagner qu'avec des combinaisons spécifiques. Cela permet en fait d'augmenter considérablement la difficulté du jeu. Par contre, je ne joue jamais à des jeux comme Texas Poker ou Zhajinhua [une variante chinoise du poker]. En fait, ce sont des jeux destinés au pari, car il n'y a pas de technique dedans. Ce qui m'intéresse dans les jeux de cartes, c'est leur caractère divertissant et non pas la possibilité de gagner de l'argent. (Enquêté, 28 ans)

Il faut pourtant apporter une nuance au propos de cet enquêté, dans la mesure où le poker, que l'enquêté considérait comme un jeu de hasard pur, est en réalité un jeu susceptible de maîtrise technique. Mais il n'en demeure pas moins que son propos permet de mettre en lumière un rapport fondamental entre l'argent et le jeu. En effet, dans ces jeux où la compétence est aussi déterminante, sinon plus, que la chance, l'argent ne constitue qu'un enjeu facultatif, dans la mesure où la mise en place de tactiques, en ellemême, peut suffire pour générer des plaisirs ludiques. Et «le désir de voir reconnue son excellence dans un domaine donné " (Caillois, 1991, p. 52) sera un second enjeu immatériel destiné au gagnant. En un mot, les multiples enjeux que possèdent les jeux susceptibles de maîtrise technique donnent aux joueurs la possibilité d'être engagés par des aspirations autres que la recherche du profit. 
r, un paradoxe semblait apparaitre, lorsque des enquêtés, tout en nous précisant qu'ils restaient des joueurs et non des parieurs, nous ont confié que les pseudo-agôn qu'ils appréciaient sur le plan ludique ne pouvaient se passer de l'argent. Ce paradoxe apparent nous amène devant une question centrale, à laquelle nous n'avons, jusqu'ici, pas encore apporté de réponse précise : comment l'argent peut-il devenir un instrument au service du ludisme?

Il faut noter qu'à cette question, les psychologues ont déjà implicitement apporté un élément de réponse en montrant un effet spécifique de l'argent sur les joueurs. Il s'agit d'une sensation d'excitation que l'argent pourra susciter chez ces derniers, et qui peut être observée au moyen de l'observation de la fréquence cardiaque, de la conductibilité cutanée ou de la pression sanguine (Inserm, 2008). Alors que ce mécanisme psychologique a initialement été mobilisé pour la compréhension de la pathologie addictive, il a été repris par Martignoni (2000), sous le concept de «libido», pour faire de l'argent un générateur de plaisir libidinal sans pour autant y voir une source de pathologie addictive. Mais à partir de notre observation sur des jeux d'argent multijoueurs et susceptibles de maîtrise technique, nous avons identifié une fonction ludique de l'argent d'un tout autre ordre.

Cette fonction renvoie à une sorte de psychologie économique, historiquement formée et démontrée par Simmel (2007), et qui consiste en une disposition de l'être humain à se féliciter d'un gain et se désoler d'une perte. À ce propos, le rapport à l'argent d'un milliardaire chinois que nous avons interrogé est assez illustratif : «Aussi riche sois-tu, c'est toujours triste de perdre de l'argent lorsque tu joues. " (Enquêté, 43 ans)

Au travers de cette disposition qui conduira quasi-systématiquement les joueurs à éviter de perdre de l'argent quel que soit le montant à jouer ${ }^{4}$, l'introduction de l'argent dans un jeu plus ou moins technique aura ainsi pour fonction de prévenir la nonchalance des joueurs, la participation active de chacun d'entre eux étant nécessaire en vue de maintenir le niveau technique du jeu ${ }^{5}$ ainsi que les enjeux immatériels qui y sont associés (ne sera-t-il pas difficile de faire preuve de sa supériorité technique, lorsque les personnes qu'on a vaincues n'avaient pas joué de manière sérieuse ?) :

On ne peut pas se passer de l'argent. Parce que s'il n'y a pas d'argent, les gens vont jouer de manière indifférente. Et cela posera un problème, parce que le jeu ne donnera, dans ce cas-là, aucun plaisir. En fait, un jeu devient très ennuyeux, à partir du moment où l'on n'a plus besoin de se creuser la tête. (Enquêté, 62 ans)

Pour terminer cette partie de démonstration, nous proposons le réexamen d'une remarque de Roger Caillois que nos résultats d'enquête semblent rejoindre. Selon ce théoricien du jeu, «En général, le rôle de l'argent est d'autant plus considérable que la part du hasard est plus grande et par conséquent la défense du joueur plus faible " (Caillois, 1991, p. 58). Mais pour argumenter cette formule, dont nous ne réfutons pas la justesse tant qu'elle ne s'applique pas à tous les jeux d'argent, il explique que «l'alea n'a pas pour fonction de faire gagner de l'argent aux plus intelligents, mais tout au contraire d'abolir les supériorités naturelles ou acquises des individus, afin de mettre chacun sur un pied d'égalité absolue devant le verdict aveugle de la chance» (Ibid.). Or, suivant ce raisonnement, deux joueurs d'échecs auraient plus de chances de parier s'ils sont aussi forts l'un que l'autre et, dans un casino, la roulette aurait recueilli plus de mises que le poker. Sauf qu'en réalité, les choses ne se passent probablement pas de cette manière.

En effet, il nous semble que ce qui distingue le plus les pseudo-agôn aptes au pari de ceux qui y sont impropres, résident moins dans le registre de la technicité que dans celle de la 
temporalité. Plus précisément, s'il est impensable que les échecs ou le go - pour lesquels on a même inventé le pendule d'échecs pour empêcher les joueurs de passer des heures à déplacer une pièce - se retrouvent un jour dans un casino, ce n'est pas tant en raison de leur quasi-imperméabilité aux effets du hasard que leur lenteur qui les rend impropres au pari. Car si le parieur est après tout (pour ne pas dire avant tout) celui qui cherche à gagner de l'argent, il n'est pas étonnant qu'il souhaite également le faire de façon accélérée. En témoignent les polémiques autour des loteries à tirage fréquent considérées comme étant excessivement addictives (Trucy, 2006 ; Turay, 2007), même si les arguments patho-logiques mobilisés dans ces polémiques dépassent largement notre approche qui ne permet que l'identification des éléments socio-logiques.

\section{Conclusion}

En mettant en évidence le double statut de l'argent du jeu, à savoir l'argent comme instrument ludique ou comme objectif final, nous ne cherchons pas à établir une frontière étanche entre jouer et parier, frontière qui pourrait s'avérer plus poreuse dans les pratiques que dans les discours. En revanche, cette séparation conceptuelle constitue une condition intéressante pour rompre avec une vision réductrice, laquelle consiste à faire du joueur un orpailleur promis à l'échec. En réalité, les joueurs ont d'autant plus «raison" de miser, que la mise se justifie avant tout par ses fonctions proprement ludiques, puisque la rationalité en question ne se mesure plus à l'aune du gain matériel uniquement réservé au gagnant, mais du plaisir ludique également accessible au perdant.

À travers l'observation des jeux d'argent, durant lesquels la recherche du profit a été réprimée pour la sauvegarde du lien social entre les participants, nous avons essayé de donner du contenu à la dimension ludique de l'argent ainsi isolée. Notre hypothèse, qui ne saurait prétendre épuiser l'ensemble des significations ludiques de l'argent, consiste à voir dans ce dernier une protection contre l'anti-jeu que constituerait la nonchalance des joueurs, et ce, tant que la figure du joueur désirant perdre (Valleur et Bucher, 1997) représente un phénomène marginal, sinon un mythe invérifiable.

\section{BIBLIOGRAPHIE}

AMADIEU T. (2011), « L'argent du jeu. Les représentations normatives relatives aux jeux de hasard », in Actes du colloque Les usages sociaux de l'argent, CENS, Juin 2011.

BENTHAM J. (1840), CEuvres de Jérémie Bentham. Tome Premier, Bruxelles, Société belge de librairie. BRODY A. (2011), « Le poker : une forme ludomonétaire de la socialisation », in Actes du colloque Les usages sociaux de l'argent, CENS, Juin 2011.

CAILLOIS R. (1991), Les jeux et les hommes : le masque et le vertige [1958], Paris, Gallimard.

CARCASSONNE G. (2011), « De quelques mystères du jeu », Pouvoirs, vol. 139, n 4, pp. 15-23.

GODELIER M. (1996), L'énigme du don, Paris, Fayard. 
INSERM (2008), Jeux de hasard et d'argent : Contextes et addictions, Inserm.

MARTIGNONI-HUTIN J.-P. (1993), Faites vos jeux, Paris, L'Harmattan.

MARTIGNONI-HUTIN J.-P. (2000), Ethno-sociologie des machines à sous : que le hasard vous serve, mais préparez-vous à l'accueillir, Paris, L'Harmattan.

MARTIGNONI-HUTIN J.-P. (2005), « Que peut apporter la sociologie dans le débat sur le jeu compulsif?", Psychotropes, vol. 11, n², p. 55-86.

NEURRISSE A. (1993), Les jeux d'argent et de Hasard : Casinos et Loteries, Paris, Hermé.

PAPINEAU E. (2000), Le jeu dans la Chine contemporaine : mah-jong, jeu de go et autres loisirs, Paris, L'Harmattan.

SIMMEL G. (2007), Philosophie de l'argent [1900], Paris, PUF.

SMITH A. H. (2006), Mœurs curieuses des chinois [1894], Chicoutimi, J.-M. Tremblay.

TESTART A. (2001), «Échange marchand, échange non marchand », Revue française de sociologie, vol. $42, \mathrm{n}^{\circ} 4$, pp. 719-748.

TRUCY F. (2006), L'évolution des jeux de hasard et d'argent : le modèle français à l'épreuve, Rapport d'information au Sénat.

TURAY S. (2007), La Française des Jeux :jackpot de l'Etat ?, Paris, Éditions Générales First, 2007.

VALLEUR M., BUCHER C. (1997), Le Jeu pathologique, Paris, PUF.

ZELIZER V. (2005), La signification sociale de l'argent [1994], Paris, Seuil.

\section{NOTES}

1. D'après Jeremy Bentham, à montant égal, l'utilité marginale du gain est toujours inférieure à la désutilité marginale de la perte. D'où l'irrationalité mathématique de participer même à un pari équitable : «Que les chances en fait d'argent soient égales, les chances en fait de bonheur sont toujours défavorables » (Bentham, 1840, p. 61).

2. Il s'agit de jeux de société régionaux dont, par exemple, le fameux mah-jong qui se joue à quatre joueurs.

3. Dans sa remarquable monographie Mours curieuses des Chinois, Arthur H. Smith (2006), un missionnaire américain ayant vécu en Chine pendant deux tiers de sa vie, a consacré tout un chapitre à la frugalité chinoise qui l'avait beaucoup impressionné.

4. À l'exception de ce qu'on peut appeler le «mah-jong professionnel ». Ce dernier désigne une situation dans laquelle un joueur cherche à offrir un pot-de-vin en se perdant volontairement son argent (Papineau, 2000).

5. À ce propos, Aymeric Brody, dans ses travaux sur les joueurs de poker (2011), a fourni des exemples concrets montrant que l'absence d'enjeu monétaire dans le poker risque d'atteindre aussi bien « l'attitude du joueur » que « la structure même du jeu ». 


\section{RÉSUMÉS}

Sans préjuger de la fonction précise de l'argent dans le jeu, l'expression « jeu d'argent » constitue pourtant un élément de brouillage pour la compréhension scientifique de l'activité qu'elle désigne : on pense si souvent qu'il s'agit d'un jeu pour l'argent qu'on tend à ignorer la possibilité d'un jeu avec l'argent. En revanche, ce double statut de l'argent du jeu est une évidence aux yeux des Chinois, en ce que leur langage courant connaît une distinction lexicale entre "parier " (pour l'argent) et « jouer» (avec l'argent). Si cette sensibilité au double statut de l'argent du jeu semble se justifier par un système éthico-juridique chinois qui condamne l'acte de " parier » tout en autorisant celui de "jouer», cet article propose une analyse des conditions sociales dans lesquelles la revendication du statut de «joueur» ne relève pas d'une simple conformité au système éthico-juridique en question.

Without prejudging the specific function of money in games, the term "gambling" (or literally translated from French, "game of money") seems to be rather confusing for the scientific understanding of the activities this term actually refers to: We consider it so often as a game for money that we tend to ignore the possibility of it being a game with money. This dual status of money in games is however obvious in the eyes of the Chinese, since in their everyday language there is a lexical distinction between "bet" (for money) and "play" (with money). This sensitivity to the distinction of the status of money in games could probably be justified by a Chinese ethical and legal system which condemns the act of "betting" whilst allowing that of "playing". This article on the other hand proposes an analysis of the social conditions in which the claim of the status of "player" is not only to comply with the ethical-legal system in question.

\section{INDEX}

Mots-clés : jeu d'argent, parier, jouer, lien social, don réciproque, enjeu

Keywords : gambling, bet, play, social link, reciprocal gift, stake

\section{AUTEUR}

\section{SHEN HU}

Université Paris Descartes 